\title{
Water Kefir: A Review of its Microbiological Profile, Antioxidant Potential and Sensory Quality
}

\section{Yuxin Cai, Anirudh Sounderrajan and Luca Serventi*}

Department of Wine, Food and Molecular Bioscience, Lincoln University, New Zealand

*Corresponding Author: Luca Serventi, Department of Wine, Food and Molecular Biosciences, Faculty of Agriculture and Life Sciences, Lincoln University, Lincoln, Christchurch, New Zealand.

\section{Received: April 28, 2020}

Published: May 27, 2020

(C) All rights are reserved by Luca Serventi., et al.

\begin{abstract}
Water kefir is a traditional beverage made from the symbiotic fermentation of kefir grains with sweetened water. Fruits such as apples, figs and kiwifruits can be used as flavouring agent or as source of fermentable sugars. Vegetables like pumpkin work similarly as fermentation substrate. This process is carried out at $25-30^{\circ} \mathrm{C}$ in anaerobic conditions, leading to a pH of $3.5-4.5$ and alcohol content below 1\%. The yeast strains mainly belong to Kluyveromyces and Saccharomyces. It is particularly rich in probiotics, such as the lactic acid bacteria Acetobacter spp., Lactobacillus acidophilus, brevis, casei, fermentum, helveticus, kefiri, kefiranofaciens parakefiri, lactis and Leuconostoc mesenteroides. In addition, yeast strains of Kluyveromyces lactis and Saccharomyces cerevisiae are typically present. These microorganisms are embedded in a matrix of kefiran, soluble fibre with hypocholesterolemic and hepatoprotective activities. Fermentation operated by kefir grains on fruit releases glutathione, organic acids and phenolic compounds, all of which act as antioxidants. Water kefir owes its distinctive sour aroma and flavour to lactic acid and alcoholic fermentation. Fruits and vegetables can provide nutrients such as amino acid, vitamins and minerals. Fermentation-induced hydrolysis of fibre releases sugars that lower kefir sourness in favour of sweeter notes.
\end{abstract}

Keywords: Fruits; Kefir; Lactic Acid Bacteria; Probiotic; Symbiotic Fermentation; Water

\section{Introduction}

Kefir is a traditional beverage made from the symbiotic fermentation of kefir grains with milk or water [1]. These grains are small, irregularly shaped granules with a cauliflower-like appearance having elastic structure which contain a mixture of lactic acid bacteria, yeast within a polysaccharide and protein matrix. It has been introduced centuries ago in the Caucasus Mountains.

Kefir is considered to promote health therefore it has been consumed extensively until now [2]. For example, researchers investigated the cells of mice fed with kefir and performed the angiogenesis assay in ex vivo. Results provided a stable evidence that water kefir can reduce the proliferation, migration and invasion of 4T1 cell, which is a breast cancer cell line derived from the mammary gland tissue of a mouse. In addition, there was a positive result in terms of angiogenesis inhibition. Hence, kefir had an anticancer effect. Besides, it also has anti-inflammatory, immunomodulation, and other properties due to its probiotic content. The definition of probiotics is live microorganisms that can confer health benefits in the host when administered in adequate amounts [3]. The grain used to ferment water kefir consisted of a variety of beneficial live microorganisms, such as bacteria and yeast. Furthermore, several of the different live microorganisms in water kefir grains have probiotic properties that exert beneficial health effects to the host when sufficiently consumed [4].

Kefir grains can ferment a matrix of milk or water. Kefir made from milk can provide antioxidant and antimutagenic activity, which are helpful to treat tuberculosis, gastrointestinal disease and cancer [5]. Nonetheless, it still has limitations. For those who follow a vegan diet and those who are lactose intolerant, alternatives to milk are needed. Therefore water kefir could be a choice. The main ingredients of water kefir are kefir grains, sugar and water. In order to improve the taste of water kefir, fresh and dried fruits were added into water kefir. The production of water kefir must take place within certain conditions, such as the fermentation temperature between 25 and $30^{\circ} \mathrm{C}$. In addition, the $\mathrm{pH}$ drops throughout the fermentation process, from the initial $\mathrm{pH}$ of about 7 to a final range of 3.0 to 4.5 [6]. The final alcohol content is typically $0.5 \%$ or below. If all of these conditions are satisfied, a good quality water kefir can be produced [7]. 
During the processing of water kefir, several bacteria and microorganisms grow. The main microorganisms are lactic bacteria (LAB) species, Lactobacillus hilgardii, Lactobacillus paracasei, Lactobacillus nagelii and the yeast species Saccharomyces cerevisiae. The flavour of the final water kefir liquor is the result of the metabolism of these bacterial and yeast [8]. Water kefir is a fermented drink rich in antioxidants. The polysaccharides produced by kefir microorganism is also called kefiran. Kefiran has a wide application in the food industry and it can be used as a thickener, stabilizer, emulsifier, fat substitute or gelling agent, and also shows antitumor activity. In a paper, the study showed that the growth and kefiran rates of Lactobacillus kefiranofaciens have a significant increase in the mixture culture (added with Saccharomyces cerevisiae). When using this type of mixed culture, several foods such as beer, water kefir and wines can be enriched. The usage of the mixture culture can enhance the growth and kefiran rates of Lactobacillus kefiranofaciens, which are useful way can be used in commercial product [9]. Another important point worth mentioning is food safety. The processing must meet the strict criteria related to quality, safety and functionality. The international food standards (Codex Alimentarius) stated a key quality criterion is that the accurately defined numbers of viable cells in kefir is a minimum of $10^{7} \mathrm{CFU} \mathrm{mL} \mathrm{m}^{-1}$ bacteria and $10^{4} \mathrm{CFU} \mathrm{mL}^{-1}$ yeasts [4].

This review aims to investigate water kefir, discussing its processing, nutritional content and factors influencing its quality.

\section{Discussion}

\section{Ingredients}

Water kefir is a naturally fermented beverage produced and drunk at household scale around the world [10]. The main ingredients include water, dried fruits, sugar and kefir grains.

Kefir grains consists of combination of numerous yeasts primarily Kluyveromyces, Candida, Saccharomyces and some species of lactic acid bacteria including Lactobacillus, Leuconostoc, Lactococcus and Streptococcus, which are embedded in a robust water soluble branched glucogalactan matrix named kefiran [11]. The existence of D-glucose and D-galactose in an equal ratio in kefiran is responsible for the association of micro-organisms in kefir grains [12]. Kefiran is viscous, water soluble and resistant to bowel enzymatic hydrolysis. Kefir grains are gelatinous and elastic resembling a small cauliflower of white colour. They have a variable size ranging from 0.3 to $3.5 \mathrm{~cm}$ in diameter. Kefir grains contain around $34 \%$ of total protein, of which $27 \%$ are insoluble protein. Kefir grains also contain around $4 \%$ of fat, $45 \%$ of mucopolysaccharide and some amount of Vitamins $\mathrm{B}$ and $\mathrm{K}$, calcium, phosphorous and magnesium. There are two major portions of kefir grains: the peripheral portion and the inner portion. The peripheral portion consists of bacteria, largely Bacillus species, while the inner por- tion contain yeasts, on the interface of these two portions, there are both bacteria and yeasts with long polysaccharide filaments. When it comes to storing kefir grains, there are several ways; refrigeration, lyophilising, vacuum drying and freezing. Kefir grains never lose their activity if they are stored under favourable conditions [12].

The fruits commonly used for water kefir fermentation processes are dried figs and lemons as well as pomegranates. Figs can provide nutrients such as amino acid, vitamins and minerals and also enhance the flavour of beverages [13]. Sometimes, other ingredients such as tea and herbs are also added depending on consumer preference [13]. Fruit juices can assist in fermentation and as carriers of probiotics since they contain high concentrations of sugars, dietary fibre and other components which are of high nutritional value, like antioxidant polyphenolics that make them easy to gain consumer acceptance [7]. Research on fruit juices from apple, orange, lemon, cranberry, pineapple and pomegranate are being carried out, among these mentioned fruits, pomegranate has shown to be the leader in the healthy beverage industry. Pomegranate contains ten percent sugars mainly comprising of glucose and fructose, some amounts of pectin, citric acids, malic acids, phenolics, flavonoids and anthocyanins [7].

\section{Process}

The industrial production process consists of three main steps: a first fermentation, a rest period at low temperature and a second fermentation. The whole process requires 2 to 4 days of anaerobic fermentation [14]. The separation of water kefir grains from water kefir liquor should take place at the end of a fermentation process through sieving. The sieving of these water kefir grains used again for a next water kefir fermentation process by backslapping [8]. Backslapping is a process that taking the aliquot of SCOBY (Symbiotic Culture of Bacteria and Yeast), such as jelly layer of yeast and bacteria, from a fermented product and add it to a new product to ferment it. It can add microorganisms from a fermented juice to a non-fermented juice.

The fermentation step is important for water kefir processing. In order to start a fermentation, an inoculum must be prepared. Typically, pre-cultivated water kefir grains are used to start a fermentation process. For example, for a batch of $100 \mathrm{ml}$ of water kefir, about $15 \mathrm{~g}$ of water kefir grains, $6 \mathrm{~g}$ of unrefined cane sugar, 85 $\mathrm{ml}$ of tap water and $5 \mathrm{~g}$ of dried figs were mixed together in a $21^{\circ} \mathrm{C}$ water bath to incubate [15]. They were backslapped, which means that adding starter culture from previous fermentation for every 72 hours. Under the same conditions, the sieving and re-cultivated the water kefir grains from the fermenting medium to a fresh medium [15]. The mass of water kefir grains increased after each backslapping procedure, yielding more than $600 \mathrm{~g}$ of water kefir [15]. Kefir 
water grains were used as inoculants for water kefir fermentation in Schott bottles. The suspension was stirred evenly and centrifuged. In order to obtain the final fig extract, the supernatant was filtered through a coffee filter. Finally, incubating the fermentation bottles in a water bath at $21^{\circ} \mathrm{C}[15]$.

The production of water kefir must take place within certain conditions, such as the fermentation temperature between 25 and $30^{\circ} \mathrm{C}$. The $\mathrm{pH}$ drops from an initial value of around 7 to $3.0-4.5$ [6]. The initial pH of fruits such as pomegranate would lower during the fermentation processes to a pH of around 3 to 5 [16]. The fermentation is stopped when the alcoholic degree had reached a value between $0.5-1 \% \mathrm{v} / \mathrm{v}$, which is acceptable for the characterization of a low-alcoholic beverage [7].

Although the process of water kefir looks easy, many problems may occur and should be solved in industrial production. The unstable fermentation process of water kefir is a main reason that limit the production of water kefir in the industry. Also, the variable production quality of the water kefir beverages cannot meet the expectations of customers who are purchasing water kefir beverages. In addition, successful backslapping of the water kefir grains in fermentation need the growth of water kefir grain. The lower growth of water kefir grain blocks the upscaling of the yields of a water kefir during the production process. Water kefir grains play a vital role in water kefir production. During the fermentation process, the grain growth stays zero because of freezing and thawing of the water kefir grains. Laureys and De Vuyst [15] indicated that water kefir grains consist of about $86 \%$ water and the growth of ice crystals during freezing process would damage the polysaccharide structure of the water kefir grains, therefore it influences fermentation of water kefir. Gulitz and collaborators [5] pointed that a more suitable technique can used for their storage, which is before drying process freezing the fresh visible water kefir grains in the liquid nitrogen quickly.

\section{Bacterial diversity}

The term probiotic means 'for life' and is derived from the Greek language, first introduced in 1965 [17]. Probiotics and fermented milk products attract the attention of researchers from various fields, such as health care, industry and pharmacy. It was showed that kefir contains dietary probiotics which have a great potential for cancer prevention and treatment. Probiotics contain live bacteria and are live microbial food supplements that beneficially affect the host by improving the microbial balance [18]. Nonetheless, probiotic microorganisms sometimes are sensitive to various physicochemical stresses such as $\mathrm{pH}$, acidity, temperature and preservatives. Kefir is one of the most popular probiotic beverages known [18]. In recent years, interest in such probiotic species has increased as more is learned about the microorganisms used in the fermentation process, and the possibility of adding beneficial bacteria to food products. In addition, more consumers are increasingly looking to improve their health and increase their resistance to disease through dietary means [19]. The starter culture used to produce this beverage has a varying and complex microbial composition that includes species of yeasts, lactic acid bacterial (LAB), acetic acid bacteria (AAB) and fungi [20]. All of these microorganisms may have a probiotic potential. Kefir is a beverage which is produced by the mixture of the diverse spectrum of microbial species. The largest portion present in water kefir are Lactobacilli, accounting for around $65-80 \%$ of the microbial population. The Lactococci and yeasts constitute the remaining portion of the microbes present in the kefir grain [20].

An elevated level of bacterial diversity was observed in kefir grains with five major bacterial phyla that were Firmicutes, Proteobacteria, Actinobacteria, Cyanobacteria and Bacteroidetes. Firmicutes and Proteobacteria were found to be the dominant phyla in kefir grains [21]. Bacterial species belonging to Actinobacteria phylum were the third highly found phylum in all grains. Cyanobacteria and Bacteroidetes phyla were recognised in kefir grains even though at low levels. Enterobacteriaceae were dominant in certain kefir grains. Other kefir grains constituted Lactobacillaceae, Moraxellaceae and Pseudomonadaceae were found in all kefir grains except for Enterococcaceae and Streptococcaceae. Lactobacilli species have a rich diversity in kefir grains. Lactobacillus kefiranofaciens is the dominant bacterial species in some grains and it constitutes half of the microbial species. Lactobacillus kefiri, Lactobacillus apis and Lactobacillus ultunensis were present in lower numbers in some of the grains, along with Enterobacter amnigenus and Enterobacter hormaechei [21]. Dertli and collaborators [22] observed that L. kefiranofaciens was the dominant lactic acid bacterial species in kefir grains. Recent studies have identified more Lactobacillus kefiranofaciens in grains that were taken from Turkey. Enterobacter amnigenus and Enterobacter hormaechei, which are usually found in cheese, were also present in all the kefir grains [22]. Pseudomonas spp. were found in some kefir grains and these species should be taken proper care of as they be harmful to human health [22].

Lactic acid bacteria (LAB) are the main type of bacteria isolated from water kefir. It includes Lactobacillus acidophilus, Lactobacillus brevis, Lactobacillus casei, Lactobacillus fermentum, Lactobacillus helveticus, Lactobacillus kefiri, Lactobacillus parakefiri, Lactococcus lactis and Leuconostoc mesenteroides [19]. Specific strains of lactic acid bacteria, especially some genera Lactobacillus, can be used as probiotics because of their ability to modulate the immune system as well as their capacity to inhibit the growth or invasion of pathogenic bacteria and parasites. Lactobacillus kefiri is a probiotic microorganism in vitro, exerting a protective action against the invasion of Salmonella enterica serovar enteritidis and are able to 
antagonize the cytotoxic effects of clostridial toxins on Vero cells [23]. The amount of lactic acid bacteria produced during the fermentation process in the fruit kefir is around 30 to $300 \mathrm{cfu} / \mathrm{ml}$ [7]. In milk kefir, the number of bacteria presented may vary from 665 to $918 \mathrm{cfu} / \mathrm{g}$, while the yeasts population is lower than bacteria (around 157 to $400 \mathrm{cfu} / \mathrm{g}$ ) [12].

Several metabolites such as diacetyl, ethanol, acetaldehyde and acetate, are formed during kefir fermentations by probiotic microorganisms and give kefir unique physical, chemical and sensory characteristics. The difference between homofermentative LAB and heterofermentative LAB is in their end-products. During fermentation, homofermentative LAB mainly produce lactic acid, while heterofermentative one produces carbon dioxide $\left(\mathrm{CO}_{2}\right)$ and lactose. In addition, the main role of the yeast in kefir grains are produced the ethanol and $\mathrm{CO}_{2}$. Acetobacter plays an important role in improving the taste and the consistency of the product, although it just comprises about $1 \%$ of the total visible counts in the grain [24].

\section{Yeast and fungal diversity}

There is a profuse yeast population that exists in symbiotic relationship with bacteria and fungi in the kefir grains. Yeast species present in all kefir grains were Rhodotorula dairenensis and Rhodotorula mucilaginosa. Yarrowia lipolytica and Cryptococcus victoriae were present in lower numbers [22]. Otles and Çağındı [25] found that two morphotypes of yeasts were dominating by using scanning electron microscopy. Most of the yeast cells found were elongated with irregular shapes showing different stigmas at the pole sides as the result of former budding events. Several studies $[26,27]$ have reported that three genera of yeasts are commonly found in kefir grains: Saccharomyces, Kluyveromyces and Candida. Numerous species of Saccharomyces have been isolated from kefir. However, S. cerevisiae and S. unisporus were more common and present in most of the varieties of kefir grains. $K$. marxianus and $K$. lactis were the only two common species of the Kluyveromyces family known for utilising lactose. The Candida family consists of an extensive range of species, with $C$. holmii and $C$. kefyr being the most dominant. Kazachstania unispora, also known as Saccharomyces unisporus, is present in kefir grains. Numerous sequencingbased methods have identified over a dozen yeast species that had not been previously been associated with kefir, such as Dekkera anomala, Issatchenkia orientalis and Pichia fermentans. Yeasts species like $S$. boulardii have shown to improve the symptoms of Clostridium difficile associated diarrhoea, by reducing inflammation and altering the immune state in the gut [28]. These yeasts can be considered as a probiotic food supplements. For example, Saccharomyces cerevisiae var. boulardii is a probiotic yeast used in various commercial formulations as therapeutic agent for the treatment of a variety of gut disorders, normalising intestinal flora [29]. Yeasts isolated from kefir grains include Kluyveromyces marxianus, Torula kefir, Saccharomyces exiguus and Candida lambica [20].

Ascomycota is the major fungal phylum in Kefir grains, followed by Basidiomycota and Zygomycota phyla. There are six family levels observed in kefir grains: Dipodascaceae, Pichiaceae, Saccharomycetaceae, Malasseziaceae, Trichosporonaceae and Mucoraceae. It was observed that yeast and Dipodascaceae species were dominant, although not every kefir grain will have the same composition of fungal species. Occasionally, mould species like Mucor circinelloides were detected. Saccharomyces cerevisiae was found to be present in low numbers. Previous studies have analysed the microflora of kefir grains using DGEE technique (Denaturing Gradient Gel Electrophoresis) and found that low levels of diversity in kefir grains mainly comprised of Saccharomyces spp., Kluyveromyces lactis, Kazachtania spp. and Candida spp. [22].

\section{Antioxidant potential}

Fermentation of sugary solutions by kefir grains can produce nutritionally relevant molecules such as polypeptides, polysaccharides and organic acids [30]. Studies have shown that the reducing power of these compounds was considered as an important indicator of its own potential antioxidant activity. Alsayadi and collaborators [30] proved that water kefir has a high ability to reduce $\mathrm{Fe}^{3+}$ / ferricyanide complex to the ferrous form. As the concentration of the tested samples increased, so did the reducing power. In addition, the reducing power of milk and soymilk were lower than kefir and fermented milk and soymilk [31]. A possible explanation is the synthesis of metabolites with superior reducing power, such as glutathione, upon fermentation. Glutathione can react with free radicals to stabilize and terminate radical chain reactions [31] showed that intracellular antioxidants sourced from the kefir microflora of soymilk kefir samples $(0: 100)$ had approximately 3 times higher reducing activity. On the other hand, the antioxidant activity may also come from the fruit added, such as kiwifruit and pomegranate juice [11]. Pomegranate juice contains several polyphenol components punicalagin isomers, ellagic acid derivatives and anthocyanins (delphinidin, cyanidin, pelargonidin 3- and 3,5-diglucosides). The existence of these polyphenol components in pomegranate and other fruits and vegetables provide redox properties. Hence, these molecules can act as reducing agent, hydrogen donor and singlet oxygen quencher. With the fermentation of lactic acid bacteria and some other microorganisms, the total content of polyphenols increases [32]. Also, organic acids and enzymes influence the antioxidant activity since enzymes increase the antioxidant capacity by releasing bound phenolics. For example, $\beta$-glycosidase can hydrolyse the complex phenolic compounds into simple type, therefore increasing the antioxidant potential [32]. Additionally, enzymes 
might hydrolyse fruit fibre, releasing more sugar for fermentation, which may lower the sourness of water kefir. Other organic acids such as citric and malic acids can be found in fruit-based kefir beverages, acting as antioxidants and antimicrobials, possibly preserving during storage [33].

\section{Kefiran}

Kefiran is the exopolysaccharides present in kefir grains. It was first discovered in 1967. Lactobacillus kefiranofaciens produces kefiran. It is a type of water-soluble polysaccharide containing equal amounts of glucose and galactose which is widely used in pharmaceutical industries. Lactose was found to be the best chemically defined carbon source for the production of kefiran by Lactobacillus sp. KPB-167B, compared to glucose and sucrose [34]. Kefiran is produced by lactic acid bacterial and is a type of water-soluble polysaccharide [34]. It is considered as a metabolite of capsular bacteria such as such as Lactobacillus kefir, Lactobacillus kefiranofaciens and Saccharomyces cerevisiae [35]. Kefiran has a branched hexa- or heptasaccharide repeating unit that is itself composed of a regular pentasaccharide unit, to which one or two sugar residues are linked.

Kefiran can deliver many benefits to humans. Researchers [34] performed experiments on rats, observing that kefiran lowered their level of serum cholesterol. Although kefiran itself cannot inhibit the absorption of cholesterol in food, it does limit enterohepatics-circulating cholesterol in the intestine, which can prevent the hepatic disorders caused by cholesterol and orotic acid. In addition, kefiran also expresses various preventive functions, it can prevent liver disorders and decrease the concentration of intestinal histamine to lower the possibility of histamine intolerance [34].

Kefiran expresses functional properties. It is a filmogenic material, bio-degradable polymers used in food packaging [36]. Kefiran contains glucose and galactose. The characteristics of watersolubility improves the viscosity and viscoelastic properties of acid milk gels. Under the low temperature, gel with viscoelastic properties can be formed. Furthermore, high yields of kefiran can be isolated from the grains in deproteinised whey. Due to these properties, kefiran can produce films with satisfactory mechanical properties and good appearance. Hence, it has excellent potential as a film-forming agent [37]. In addition, it can be used as a thickener, stabilizer, emulsifier, fat substitute or gelling agent, and also shows antitumor activity. Kefiran is regarded safe. There is evidence showing its antibacterial, anti-fungal and anti-inflammatory activities. Furthermore, it can be used as cicatrizing agents against a variety of infections and as a stress reducing food component due to an enhancing effect on the production of interferon $\beta$ cortisol and noradrenaline in human cell [34].

\section{Sensory quality}

The symbiotic metabolic activity of a number of bacterial and yeast species that form part of the original natural starter-kefir grains give water kefir unique flavour and aroma. Water kefir owes its distinctive flavour to being a self- carbonated product with ongoing lactic acid and alcoholic fermentations. It produces lactic acid, $\mathrm{CO}_{2}$, ethanol and other flavour-forming products [36]. Kefir is an alcoholic beverage with soured, frothy and mildly acid taste [29]. Water kefir is a non-dairy kefir produced by sucrose with or without fruit and fermented by kefir grains. The fermentation of such substrates, which are kefir grain, fruit or fruit juice, and molasses or sugar solutions, provides acidic taste, refreshing, slightly carbonated, low alcoholic and acetic notes [11]. Some fruits added to kefir improved its acceptability, being rich in sugar, proteins, amino acids, vitamins and minerals [11]. Fruits commonly used for water kefir fermentation processes are dried figs, lemons and pomegranates. Figs can provide nutrients such as amino acid, vitamins and minerals and also enhance the flavour of beverages [13]. Sometimes, other ingredients such as tea and herbs are also added depending on consumer preference [13]. Cocoa pulp-basic kefir is a new beverage made with cocoa. It is low alcohol, with acidic, refreshing, slightly carbonated taste, which due to the fermentation of substrates such as lactic acid bacteria and cocoa [33]. Pomegranate juice was used to produce fruit-based kefir [16]. It can release some organic acids such as lactic and acetic acids. All of these compounds can bring health benefits as well as changing the taste of kefir, hence improving the sensory acceptable of consumers [7,16,32]. Also, pomegranate contains glucose and fructose, which can neutralize the sour taste of kefir, thus gaining more consumer acceptance [7]. Finally, a vegetable, pumpkin, was used to develop a novel water kefir [4]. Fermentation can prolong the shelf life of pumpkin as well as changing its original taste to a sour one. Sensory analysis assessed that the lower pumpkin puree concentrations were accepted by panellists [4].

\section{Conclusion}

Water kefir is a variation of the more commonly known milk kefir, particularly suitable to those who do not consume milk due to lactose intolerance or dietary patterns such as plant-based and vegan diets. This beverage is made from the symbiotic fermentation of water sweetened with fruits and/or sugars, performed by kefir grains. Such grains contain yeasts and bacteria embedded in a polysaccharide matrix called kefiran. This soluble fibre can lower cholesterol absorption and histamine intolerance, as well as preventing liver damage. In addition, it contributes to viscosity and mouthfeel. Water kefir has antioxidant potential due fruit phenolic compounds, as well as the synthesis of glutathione and oxygen-reactive organic acids occurring upon fermentation. The fruit added to water kefir delivers water, vitamins, sugar, proteins, and amino 


\begin{tabular}{|c|c|c|}
\hline Nutrients & Properties & References \\
\hline \multicolumn{3}{|l|}{ Bacteria } \\
\hline Acetobacter spp. & Consistency of the products & 23 \\
\hline Lactobacillus kefiranofaciens & Kefiran production & 1,32 \\
\hline Lactobacillus kefiri & $\begin{array}{l}\text { Immune system modulation to inhibit the growth of } \\
\text { pathogenic bacteria and parasites }\end{array}$ & 1,24 \\
\hline $\begin{array}{l}\text { Lactobacillus acidophilus, brevis, casei, fermentum, helveticus, } \\
\text { kefiri, parakefiri, lactis; Leuconostoc mesenteroides }\end{array}$ & Lactic acid bacteria & 19 \\
\hline \multicolumn{3}{|l|}{ Yeast } \\
\hline Kluyveromyces Lactis & $\begin{array}{l}\text { Respiratory catabolism (Pasteur effect): favouring } \\
\text { respiration over alcoholic fermentation }\end{array}$ & 26 \\
\hline Saccharomyces cerevisiae & Sugar conversion in carbon dioxide and ethanol & 27 \\
\hline \multicolumn{3}{|l|}{ Fibre } \\
\hline $\begin{array}{l}\text { Kefiran (Water-soluble polysaccharide of glucose and galac- } \\
\text { tose) }\end{array}$ & Hypocholesterolemic and hepatoprotective & 32,34 \\
\hline \multicolumn{3}{|l|}{ Antioxidants } \\
\hline Glutathione & $\begin{array}{l}\text { Reaction with free radicals to stabilise and termi- } \\
\text { nate radical chain reactions }\end{array}$ & $28-29$ \\
\hline Organic acids & Antioxidants and antimicrobials & 28,31 \\
\hline Phenolic compounds & $\begin{array}{c}\text { Reducing agents, hydrogen donors and singlet } \\
\text { oxygen quenchers }\end{array}$ & 16 \\
\hline
\end{tabular}

Table 1: Nutritional properties of water kefir.

acids, thus resulting in low sour taste (typical of milk kefir) due to the enzymatic release of sugars from fibre during fermentation.

\section{Acknowledgements}

Authors acknowledge Lincoln University (Christchurch, New Zealand) for supporting the Bachelor course "FOOD 398 Design or Research Essay" and the Master course "FOOD 698 Design or Research Essay". A grateful thank you goes to Dr Yu Zhang (Children's Hospital of Pittsburgh) for transmitting his passion for research to the corresponding author. Crucial acknowledgments go to Lindy Choo, who inspired our research on water kefir.

\section{Conflict of Interest}

Authors declare no conflict of interest for this manuscript.

\section{Bibliography}

1. Fiorda Fernanda Assumpção., et al. "Microbiological, biochemical, and functional aspects of sugary kefir fermentationA review". Food Microbiology 66 (2017): 86-95.

2. Zamberi Nur Rizi., et al. "The antimetastatic and antiangiogenesis effects of kefir water on murine breast cancer cells". Integrative Cancer Therapies 15.4 (2016): NP53-NP66.
3. Ganguly NK., et al. "ICMR-DBT guidelines for evaluation of probiotics in food". The Indian Journal of Medical Research 134.1 (2011): 22.

4. Koh Wee Yin., et al. "Development of a novel fermented pumpkin-based beverage inoculated with water kefir grains: a response surface methodology approach". Food Science and Biotechnology 27.2 (2018): 525-535.

5. Gulitz Anna., et al. "The microbial diversity of water kefir". International Journal of Food Microbiology 151.3 (2011): 284288.

6. Martínez-Torres Abigail., et al. "Inferring the role of microorganisms in water kefir fermentations". International Journal of Food Science and Technology 52.2 (2017): 559-571.

7. Kazakos Stavros., et al. "Production of low-alcohol fruit beverages through fermentation of pomegranate and orange juices with kefir grains". Current Research in Nutrition and Food Science Journal 4.1 (2016): 19-26.

8. Laureys David., et al. "Investigation of the instability and low water kefir grain growth during an industrial water kefir fermentation process". Applied Microbiology and Biotechnology 101.7 (2017): 2811-2819. 
9. Cheirsilp Benjamas., et al. "Kinetic modeling of kefiran production in mixed culture of Lactobacillus kefiranofaciens and Saccharomyces cerevisiae". Process Biochemistry 42.4 (2007): 570-579.

10. Pothakos Vasileios, et al. "Acetic acid bacteria in fermented food and beverage ecosystems". Acetic Acid Bacteria. Springer, Tokyo (2016): 73-99.

11. Randazzo Walter., et al. "Development of new non-dairy beverages from Mediterranean fruit juices fermented with water kefir microorganisms". Food Microbiology 54 (2016): 40-51.

12. Rosa Damiana D., et al. "Milk kefir: nutritional, microbiological and health benefits". Nutrition Research Reviews 30.1 (2017): 82-96.

13. Adachi O., et al. "Acetic acid bacteria: ecology and physiology". Eds. Matsushita K, Toyama H, Tonouchi N, and Okamoto-Kainuma A. Springer Japan, Tokyo (2016): 273-297.

14. Laureys David, et al. "Bifidobacterium aquikefiri sp. nov., isolated from water kefir". International Journal of Systematic and Evolutionary Microbiology 66.3 (2016): 1281-1286.

15. Laureys David and Luc De Vuyst. "Microbial species diversity, community dynamics, and metabolite kinetics of water kefir fermentation". Applied and Environmental Microbiology 80.8 (2014): 2564-2572.

16. Sabokbar Nayereh and Faramarz Khodaiyan. "Characterization of pomegranate juice and whey based novel beverage fermented by kefir grains". Journal of Food Science and Technology 52.6 (2015): 3711-3718.

17. Schrezenmeir Jürgen and Michael de Vrese. "Probiotics, prebiotics, and synbiotics-approaching a definition". The American Journal of Clinical Nutrition 73.2 (2001): 361s-364s.

18. Sharifi Mohammadreza., et al. "Kefir: a powerful probiotics with anticancer properties". Medical Oncology 34.11 (2017): 183.

19. Farnworth Edward R. "Kefir-a complex probiotic". Food Science and Technology Bulletin 2.1 (2006): 1-17.

20. Witthuhn RC., et al. "Characterisation of the microbial population at different stages of kefir production and kefir grain mass cultivation". International Dairy Journal 15.4 (2005): 383-389.
21. Waldherr Florian W., et al. "Identification and characterization of a glucan-producing enzyme from Lactobacillus hilgardii TMW 1.828 involved in granule formation of water kefir". Food Microbiology 27.5 (2010): 672-678.

22. Dertli Enes and Ahmet Hilmi Çon. "Microbial diversity of traditional kefir grains and their role on kefir aroma". LWT-Food Science and Technology 85 (2017): 151-157.

23. Kesenkaş H., et al. "Kefir". Fermented foods in health and disease prevention”. Academic Press (2017): 339-361.

24. Carasi Paula., et al. "Safety characterization and antimicrobial properties of kefir-isolated Lactobacillus kefiri". Bio Med Research International (2014).

25. Otles Semih and Ozem Cagindi. "Kefir: a probiotic dairy-composition, nutritional and therapeutic aspects". Pakistan Journal of Nutrition 2.2 (2003): 54-59.

26. Rodicio Rosaura and Jürgen J Heinisch. "Yeast on the milky way: genetics, physiology and biotechnology of Kluyveromyces lactis". Yeast 30.5 (2013): 165-177.

27. Wang Qi-Ming., et al. "Surprisingly diverged populations of Saccharomyces cerevisiae in natural environments remote from human activity". Molecular Ecology 21.22 (2012): 54045417.

28. Bourrie Benjamin CT., et al. "The microbiota and health promoting characteristics of the fermented beverage kefir". Frontiers in Microbiology 7 (2016): 647.

29. Diosma Gabriela., et al. "Yeasts from kefir grains: isolation, identification, and probiotic characterization". World Journal of Microbiology and Biotechnology 30.1 (2014): 43-53.

30. Alsayadi Muneer., et al. "Antioxidant potency of water kefir". The Journal of Microbiology, Biotechnology and Food Sciences 2.6 (2013): 2444.

31. Kesenkaş HARUN. "Antioxidant properties of kefir produced from different cow and soy milk mixtures". Journal of Agricultural Sciences 17.3 (2011).

32. Sabokbar Nayereh and Faramarz Khodaiyan. "Total phenolic content and antioxidant activities of pomegranate juice and whey based novel beverage fermented by kefir grains". Journal of Food Science and Technology 53.1 (2016): 739-747.

33. Puerari Cláudia., et al. "New cocoa pulp-based kefir beverages: Microbiological, chemical composition and sensory analysis". Food Research International 48.2 (2012): 634-640. 
34. Dailin Daniel Joe., et al. "Bioprocess development for kefiran production by Lactobacillus kefiranofaciens in semi industrial scale bioreactor". Saudi Journal of Biological Sciences 23.4 (2016): 495-502.

35. Pop Carmen., et al. "Fingerprint profiling of polysaccharide kefiran extracted from kefir grains biomass". Journal of Agroalimentary Processes and Technologies 21.2 (2015): 207-212.

36. Shahabi-Ghahfarrokhi Iman, et al. "Effect of $\gamma$-irradiation on the physical and mechanical properties of kefiran biopolymer film". International Journal of Biological Macromolecules 74 (2015): 343-350.

37. Motedayen Ali Akbar., et al. "Development and characterisation of composite films made of kefiran and starch". Food Chemistry 136.3-4 (2013): 1231-1238.

38. Beshkova DM., et al. "Production of volatile aroma compounds by kefir starter cultures". International Dairy Journal 13.7 (2003): 529-535.

\section{Assets from publication with us}

- Prompt Acknowledgement after receiving the article

- Thorough Double blinded peer review

- Rapid Publication

- Issue of Publication Certificate

- High visibility of your Published work

Website: https://www.actascientific.com/

Submit Article: https://www.actascientific.com/submission.php Email us: editor@actascientific.com

Contact us: +919182824667 